\title{
Perioperative Coagulopathy in Patients with Normal Preoperative Coagulation Screen: A Couple of Coagulopathic Clinical Case Conundrums
}

\author{
Joseph N. Monteiro ${ }^{1}$ Shwetal Goraksha ${ }^{1}$ Ninad S. Dhokte ${ }^{1}$ Balkrishna Padate ${ }^{2}$ \\ 1Department of Anaesthesiology, P. D. Hinduja Hospital and \\ Medical Research Centre. Mumbai, Maharashtra, India \\ 2 Department of Hematology, P. D. Hinduja Hospital and Medical \\ Research Centre. Mumbai, Maharashtra, India \\ Address for correspondence Joseph N. Monteiro, MD, Department \\ of Anaesthesiology, P. D. Hinduja Hospital and Medical Research \\ Centre. Mumbai, Maharashtra 400016, India \\ (e-mail: monteiro04@gmail.com).
}

J Neuroanaesthesiol Crit Care 2021;8:144-146.

\begin{abstract}
Keywords

- thromboelastography

- thrombin activatable fibrinolysis inhibitor

- selective serotonin reuptake inhibitor

Perioperative coagulopathy impacts patient outcome by influencing intraoperative hemodynamics and blood loss. We present two cases which despite having normal preoperative coagulation profiles and normotension during the surgery had intraoperative coagulopathy and unusual bleeding. These cases required a multidisciplinary approach to manage them successfully. A thorough knowledge of the coagulation cascade along with a high-degree of suspicion and early recognition of coagulation is required. Abnormalities are critical for a timely intervention. Point-of-care tests like thromboelastography (TEG) as well as platelet function studies helped us arrive at an early diagnosis and initiate prompt treatment.
\end{abstract}

\section{Introduction}

Perioperative coagulopathy impacts patient outcome by influencing intraoperative hemodynamics, blood loss, and transfusion requirements. The recognition of preexisting hemostatic disturbances, basic understanding of the principles of hemostasis, and dynamic changes of hemostasis during surgery are necessary for safe patient management. The usual causes of perioperative bleeding are either trauma, surgical procedure, preoperative anticoagulant therapy, or perioperative coagulopathies.

The viscoelastic coagulation studies confirm the central role fibrinogen plays in stable clot formation as well as provide essential knowledge about changes during blood loss and fluid administration.

The newly developed cellular model of coagulation not only facilitates the understanding of coagulation but also emphasizes the importance of the tissue factor-bearing cell and activated platelets. However, several questions such as critical thresholds for fibrinogen and platelets, and impact of Factor XIII and TAFI (thrombin activatable fibrinolysis inhibitor), remain unanswered and need to be investigated further. ${ }^{1}$

Published online June 1, 2020
DOI https://doi.org/ $10.1055 / \mathrm{s}-0040-1712855$ ISSN 2348-0548.
In our recent clinical experience, we came across two cases which presented with intraoperative coagulopathy and bleeding in spite of normotension and a normal preoperative coagulation profile, as demonstrated by conventional coagulation tests (CCTs) done routinely in our institution. These cases presented a clinical conundrum which required a multidisciplinary involvement to manage them successfully.

\section{Description of Cases}

\section{Case 1}

A 45-year-old lady was posted for endonasal decompression of pituitary macroadenoma. Preoperatively, all routine investigations were within normal limits, including a normal coagulation profile and platelet count. During surgery, the patient started bleeding from operative site in spite of normal blood pressure. An intraoperative thromboelastography (TEG) was done ( - Fig. 1) which showed an increased R (reaction time), time (27.5 $\mathrm{min}$ ) and $\mathrm{K}$ time ( 8.2 minute), decreased $\alpha$ angle 26.56, and MA (maximum amplitude) decreased to $40.5 \mathrm{~mm}$, which was suggestive of decreased fibrinogen and clotting factor as well as decreased platelets' function. The total 


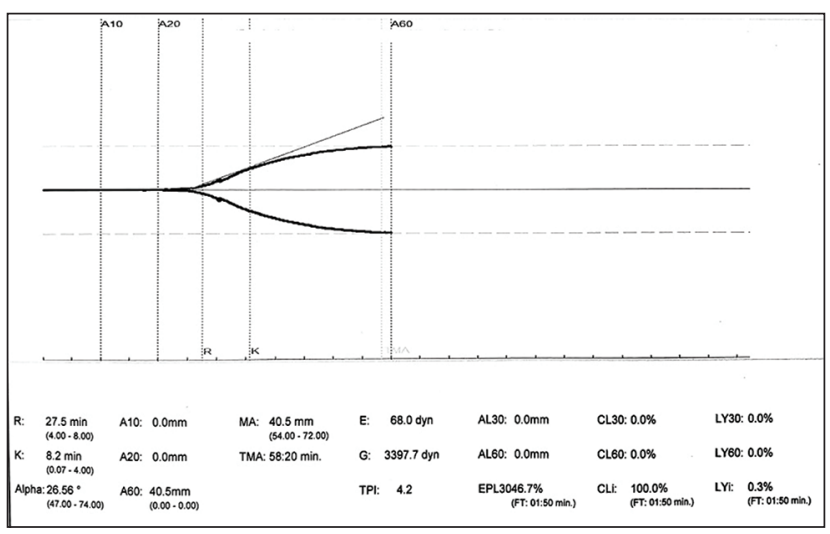

Fig. 1 Thromboelastograph Case 1.

intraoperative blood loss was around $1000 \mathrm{~mL}$; however, the patient was tachycardic and the blood pressure reduced by 10 to $15 \%$ from the baseline. The hematologist was consulted, and as per his advice, the patient was transfused with three units of packed RBCs (red blood cells), four units of FFP (fresh frozen plasma), four units of platelets, and two units of cryoprecipitate. Because of ongoing bleeding, she also received local application of recombinant factor VIIa, as advised by the hematologist. After the transfusions were completed, TEG and CCTs were repeated, and all parameters were reported to be normal. Surgery was completed successfully without any postoperative blood loss.

\section{Case 2}

A 55-year-old lady suffering from hypothyroidism and depression and was taking thyroxine tablets and selective serotonin reuptake inhibitors (SSRI) was posted for endonasal decompression of pituitary macroadenoma. The patient withheld the history of depression and a SSRI intake, which was later admitted by her relatives on questioning them intraoperatively by the hematologist due to intraoperative coagulopathy. All preoperative investigations such as the CCTs, including the coagulation profile and platelet count, were normal.

After local infiltration with lignocaine and adrenaline, and dissection of the sphenoid, the patient started bleeding profusely in spite of normal blood pressure. TEG study ( - Fig. 2) revealed an increased $\mathrm{R}$ (16.8 $\mathrm{min})$ time and $\mathrm{K}$ (4.8 $\mathrm{min}$ ) time, and decreased MA (35.7 mm), which was suggestive of clotting factor deficiency and low-platelet function. At the same time, a blood sample was sent for repeat laboratory coagulation tests as well as platelet function studies. The conventional coagulation tests are neither real-time nor as convenient as point-of-care tests, which reported abnormal platelet aggregation. SSRIs are known to lead to platelet dysfunction. The surgery was postponed and SSRIs were stopped. The risks of stopping the SSRIs, and the need to do the surgery within the same admission, as requested by the patient's relatives, were balanced; after a consensus between the psychiatrist, hematologist, neurosurgeon, and neuroanesthesiologists, it was decided to take up the patient for surgery 5 days after stopping the antidepressants. The platelet function studies and TEG

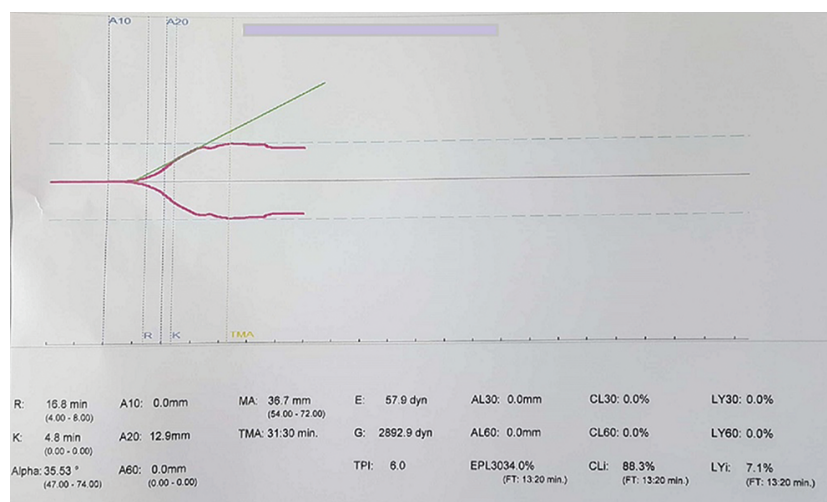

Fig. 2 Thromboelastograph Case 2.

were performed again and were reported to be normal. The patient was then operated successfully without any perioperative bleeding problems.

\section{Discussion}

Acquired coagulopathy can develop in patients with trauma, during obstetrics complications, and during major surgery, as a result of excessive blood loss and consumption of coagulation factors as well as hemodilution. In the scenario of intraoperative nonsurgical bleeding, a coagulation screen, which includes platelet count, prothrombin time (PT), activated partial thromboplastin time (aPTT), thrombin time (TT) and fibrinogen level, is useful as a guide to further management. Abnormalities in clotting results can suggest the defect in coagulation pathway as well as the type of blood product and factor concentrates necessary to stop bleeding. Coagulation disorders with a normal PT and aPTT include platelet function disorders, von Willebrand disease (vWD), factor XIII deficiency, and disorders of fibrinolytic system. ${ }^{1}$

In patients undergoing neurosurgery, the initial brain damage triggers the release of various stress factors such as tissue factor (TF), thrombin-releasing factors, and TF containing microparticles. This initiates the coagulation process which is augmented and continued by secondary brain ischemic and hemorrhagic injuries and may result in coagulopathy. ${ }^{2}$

This, when associated with hypoperfusion or endothelium damage, causes thrombomodulin release and stimulation of protein $C$ pathway, leading to the inhibition of activated cofactors $\mathrm{V}$ and VIII, decreased thrombin generation, and decrease in fibrin formation. ${ }^{3,4}$

Nonetheless, a distinct vascular microanatomy in the brain creates several regulatory points that may make contact with the hemostatic system in blood and may become involved in disease. This also triggers the amplification phase of the coagulation cascade, leading to the deposition of fibrin matrix, clots, platelets, growth factors, and enzymatic activities within the intravascular and perivascular microenvironment. Usually this is followed by clot resolution, but this process may be prolonged in chronic disease states such as tumor formation, resulting in vascular occlusion, ischemia, thrombin-mediated 
neurotoxicity, and cellular (noncoagulant) effects exerted by mediators of coagulation and fibrinolysis. , $^{5}$

Neither of our cases had consumptive coagulopathy or fibrinolysis. Case 1 possibly had one of the clotting deficiencies which still maintain normal baseline PT, INR, aPTT, and fibrinogen levels such as factor XIII deficiency, abnormal platelet function disorders, mild Von Willebrand disease or disorders of the fibrinogen system. Although these disorders have normal baseline clotting factors, they may have variable changes in TEG and can bleed only on the stimulus of minor or major surgical procedures. These changes get worse because of intraoperative bleeding and release of tissue factor from neuromanipulation. The TEG findings are not specific to any of these disorders. These patients need to follow-up postoperatively to further investigate the etiology, many of which may be inherited as well. Unfortunately, neither of our patients had yet followed-up for further evaluation.

Various drugs affect platelet aggregation, and some more specifically, due to their action on serotonin reuptake. In one of our cases (Case 2), SSRIs were used to treat depression. Both the beneficial and adverse effects of SSRIs are linked to their inhibitory action on the serotonin reuptake transporter (5-HTT). Platelets contain almost 99\% of whole blood serotonin. When there is a vascular injury, serotonin is released, resulting in the amplification of platelet aggregation and thrombus formation. Cellular serotonin is decreased by more than $80 \%$ in patients using SSRIs. This causes decreased exposure of activation proteins at the surface of platelets and impairment of platelet aggregation. Since the abrupt discontinuation of SSRIs can lead to a discontinuation syndrome (irritability, anxiety, and agitation) in patients, slow tapering is recommended for elective, nonemergency cases, a minimum of two weeks prior to the procedure. $^{7}$

A point-of-care test which helped us the management of our cases is TEG. This is a viscoelastic hemostatic assay that measures the global viscoelastic properties of whole blood clot formation under low-shear stress. It shows the dynamics of the interaction of platelets with the coagulation cascade (clot development, stabilization/strength, and fibrinolysis). However, it does not necessarily correlate with blood tests such as international normalized ratio (INR), APTT, and platelet count. ${ }^{8}$
With the knowledge of the principles of the tests used for the initial coagulation screen, and the awareness of the rare coagulation disorders, a systematic approach can be applied in the management of such patients.

\section{Conclusion}

Although our knowledge of perioperative coagulopathy has improved over the last few decades, several questions still remain unanswered. A high-degree of suspicion and early recognition of coagulation abnormalities is critical for a timely intervention. Point-of-care tests like TEG help in diagnosis. A multidisciplinary approach including a consultation with a hematologist helps in early resolution of the coagulopathy. Future studies and research are needed with a focus on characterizing clinical, biological, and genetic markers that may help identify patients at greater risk of perioperative bleeding.

\section{Conflict of Interest}

None declared.

\section{References}

1 Mya T, Heng N. An approach to the patient with non-surgical bleeding and a normal coagulation screen. Proceedings of Singapore Healthcare. 2014;23(1):21-27

2 Simmons JW, Powell MF. Acute traumatic coagulopathy: pathophysiology and resuscitation. Br J Anaesth 2016;117(suppl 3): iii31-iii43

3 Gerlach R, Krause M, Seifert V, Goerlinger K. Hemostatic and hemorrhagic problems in neurosurgical patients. Acta Neurochir (Wien) 2009;151(8):873-900

4 Goh KY, Poon WS, Chan DT, Ip CP. Tissue plasminogen activator expression in meningiomas and glioblastomas. Clin Neurol Neurosurg 2005;107(4):296-300

5 D'Asti E, Fang Y, Rak J. Brain neoplasms and coagulationlessons from heterogeneity. Rambam Maimonides Med J 2014; 5(4): e0030

6 del Zoppo GJ, Izawa Y, Hawkins BT. Hemostasis and alterations of the central nervous system. Semin Thromb Hemost 2013;39(8):856-875

7 Roose SP, Rutherford BR. Selective serotonin reuptake inhibitors and operative bleeding: a review of literature. J Clin Psychopharmacol 2016;36(6):704-709

8 Salem AM, Roh D, Kitagava RS, Choi HA, Chang TR. Assessment and management of coagulopathy in neurocritical care. Journal of NeuroCritical Care 2019;12(1):9-19 